\title{
The observation of the Earth tide is irrelevant to the speed of gravity
}

\author{
HUANG ChaoGuang \\ Theoretical Physics Center for Science Facilities, Institute of High Energy Physics, Chinese Academy of Sciences, Beijing 100049, China
}

Received March 13, 2013; accepted May 28, 2013

Recently, the observations on the earth tide at Shiquanhe and Wushi were used to show that gravity propagates at the speed of light. Based on the brief review of general relativity and the Newtonian gravitational theory, we re-analyze the experiments and cannot reach the same conclusion from the observations.

Earth tide, speed of gravity, apparent position, phase lag

Citation: Huang C G. The observation of the Earth tide is irrelevant to the speed of gravity. Chin Sci Bull, 2013, 58: 3291-3294, doi: 10.1007/s11434-013-5997-6

It is well-known that gravitational waves in general relativity (GR) will propagate at the speed of light but the stationary fields do not propagate in both GR and in the Newtonian theory. Recently, an experimental group led by Tang claims [1] that the observations of the earth tide induced by the Sun show that gravity propagates at the speed of light.

The raw observation data used in the analysis [1] is recorded by the PET gravimeters with rubidium-clocks from the Shiquanhe station in Tibet and the Wushi station in Xingjiang, which are both very far away from oceans so that the influences of ocean tides to the Earth tide are negligible. After filtered out high frequency $(v \geqslant 0.01 \mathrm{~Hz})$ disturbances, deducted a phase lag of $37 \mathrm{~s}$ which is due to the anelasticity of the Earth and averaged from $24 \mathrm{~s}$ of Arabelos [2] and $40 \mathrm{~s}$ of Ray et al. [3], and made a suitable normalization, they constructed an observation curve of the quasi-solar earth tide by subtracting the theoretical values of the lunar tides according to the Newton's law from the normalized and corrected observational data. They claimed that the observation curve lags about $500 \mathrm{~s} \approx R_{\odot} / c$ behind the curve determined by the classical Newtonian formula for tides, which indicates that gravity propagates from the Sun to the Earth with the speed of light, where $R_{\odot}$ is the mean earth-sun distance and $c$ is the speed of light in vacuum.

The purpose of the letter is to explain why GR and the Newtonian gravitational theory do not support the claim and

$\overline{\text { email: huangcg@ihep.ac.cn }}$ to present our alternative analysis of the observations reported in [1].

In GR, a gravitational field is described by geometry

$$
\mathrm{d} s^{2}=g_{\mu \nu}\left(x^{\lambda}\right) \mathrm{d} x^{\mu} \mathrm{d} x^{\nu} .
$$

A gravitational field is said to be stationary if the metric (1) permits an asymptotically timelike Killing vector. Usually, the metric of a stationary field is independent of the time coordinate $t$, namely, $g_{\mu \nu}=g_{\mu \nu}\left(x^{i}\right)$. Furthermore, if $g_{0 i}=g_{i 0}=0$, the spacetime is static, or the gravitational field is static. The gravitational field of the Sun in the heliocentric system may be described very well by Schwarzschild geometry,

$$
\mathrm{d} s^{2}=\left(1-\frac{2 G M_{\odot}}{c^{2} r}\right) c^{2} \mathrm{~d} t^{2}-\frac{\mathrm{d} r^{2}}{1-\frac{2 G M_{\odot}}{c^{2} r}}-r^{2} \mathrm{~d} \Omega^{2},
$$

where $G$ is the Newtonian gravitational constant, $M_{\odot}$ is the mass of the Sun, and $\mathrm{d} \Omega^{2}$ is the line-element on a unit sphere. In the spacetime, both the geodesic equation and the geodesic deviation equation are independent of $t$. Clearly, the interaction between the test particles and the gravitational field of the Sun has nothing to do with the propagation of gravity at a certain speed because the field is static. From the point of view of an observer in the heliocentric system, the interaction is also irrelevant with the instantaneous interaction because the gravitational field is always there and the test particles interact with the field locally. 
Strictly speaking, when GR is applied to settle the tide problem on the Earth, one should consider the perturbations on the Schwarzschild metric due to the motion of the Earth, the Moon and other planets, which make the gravitational field deviate from the static one. When the Earth is not treated as a test particle, both the Sun and the Earth will rotate around the center of mass of the Sun-Earth system if the effects of other planets and satellites are ignored. The gravitational field of the system may still be approximated as a stationary one, because the gravitational radiation from the system is extremely low $(\sim 200 \mathrm{~W})$ and because the period and the relative amplitudes of both the Earth and the Sun around the center of mass are a year and about $10^{-6}$ or less, respectively, when the eccentricities are taken into account, which is not the effect of the experiment tests. Hence, there is no propagation problem in this case. Furthermore, the gravitational field becomes non-stationary if the motions of other planets and satellites are taken into account. The non-stationary parts can be treated as the perturbations on the stationary geometry. These perturbations should spread from the sources of the perturbations but not spread from the Sun. For example, the perturbation due to the motion of the Moon should spread from the Moon, which is obviously irrelevant to the distance between the Sun and the Earth. In addition, the Earth has a finite radius and rotates around its axis, which also makes the measurement apparatus deviate from a free test particle. Again, the perturbations and deviations cannot provide any information for the propagation of gravity from the Sun to the Earth. On the other hand, in the analysis of Tang et al. [1], the characteristic properties of the perturbations to the solar gravitational field from the motion of the Moon and other planets are not taken into account except subtracting the lunar tides according to the Newton's law from the observation data. Therefore, if the propagation effect claimed by Tang et al was real, GR would be incorrect, though GR does predict that the changes in gravitational fields propagate with the speed of light.

In fact, the Newtonian treatment is enough in the discussion of the earth tides. This is because the post-Newtonian terms of the solar and lunar gravitational field are $\frac{G M_{\odot}}{c^{2} R_{\odot}} \sim$ $\frac{v_{\oplus}^{2}}{c^{2}} \sim \frac{G M_{\supset}}{c^{2} \bar{r}_{\supset}} \sim \frac{v_{\supset}^{2}}{c^{2}} \sim 10^{-8}$ times smaller than the Newtonian terms, where $M_{\supset}$ is the mass of the Moon, $\bar{r}_{\supset}$ is the mean earth-moon distance, $v_{\oplus}$ and $v_{\supset}$ are the orbital speeds of the Earth and the Moon, respectively. They are far beyond the precision of the experiments even though the superconducting gravimeters are used as in [4-6].

In Newtonian theory, the space and time are flat and absolute. In the heliocentric system, the gravitational field strength of the Sun is governed by the Newton's inversesquare law and the field strength that the Sun produces the tide on the surface of the Earth is

$$
\boldsymbol{g}_{\text {tide }}=G M_{\odot}\left(\frac{\boldsymbol{R}_{\odot}}{\boldsymbol{R}_{\odot}^{3}}-\frac{\boldsymbol{R}_{\odot}+\overline{\boldsymbol{r}}}{\left|\boldsymbol{R}_{\odot}+\overline{\boldsymbol{r}}\right|^{3}}\right),
$$

where $\boldsymbol{r}$ is the position vector in the heliocentric system, and a letter with an overbar, such as, $\overline{\boldsymbol{r}}$, denotes the position vector or coordinates in the geocentric system, and $\boldsymbol{R}_{\odot}$ is the position vector of the center of the Earth in the heliocentric system. These expressions do not depend on time $t$ explicitly and do not have the retarded expressions as expected in [1]. Therefore, they have nothing to do with the propagation at a certain speed, again. As in GR, the expressions are also irrelevant with the instantaneous interaction because the gravitational field has always been there and the interaction between test particles and the field may be interpreted locally. Even if using the point-to-point interaction analysis as did in [1] instead of the field analysis, one cannot obtain the conclusion in the heliocentric system that gravity propagates at a certain speed. The reason is very simple. In the heliocentric system, the position of the Sun is unchanged but the position of the Earth is changing. The apparatus for the gravimetric observation is fixed on the surface of the Earth and moves with the Earth, the relative velocity between the apparatus and the surface of the Earth is zero. Hence, one cannot get the conclusion of the lapse of $R_{\odot} / c$.

Since the analyses from different viewpoints show that the gravitational phenomenon in a static field is irrelevant with the propagation of gravity, how are the gravimetric observations in [1] explained?

First of all, the basic equation of the gravitational field that the Sun produces the tide in Newtonian theory, eq. (3), is modified in [1] as

$$
\boldsymbol{g}_{\text {tide }}(O, t)=-\frac{G M_{\odot}}{r_{*}^{3}\left(t_{*}\right)} \boldsymbol{r}_{*}\left(t_{*}\right)+\frac{G M_{\odot}}{R_{\odot *}^{3}\left(t_{*}\right)} \boldsymbol{R}_{\odot *}\left(t_{*}\right),
$$

where $\boldsymbol{r}$ and $\boldsymbol{R}_{\odot}$ are the position vectors of the apparatus and the center of the Earth in the heliocentric system, respectively. Again, $t_{*}=t-R_{\odot} / c$ is called the retarded time. The subscript $*$ in $\boldsymbol{r}$ and $\boldsymbol{R}_{\odot}$ indicates that the apparent positions are taken. Eq. (4) is referred to as the practical Newtonian formula (PNF) in [1]. But, where does it come from? The authors claim that since the apparent positions are used in the celestial coordinate system and geographical coordinate system $[7,8]$, the geometric positions on the right hand side of eq. (3) should be replaced by their apparent positions.

In [8], however, the standard Newtonian formula,

$$
V_{\text {tot }}=\frac{G M_{\odot}}{R_{\odot}} \frac{1}{\sqrt{1+\left(\bar{r}_{\oplus} / R_{\odot}\right)^{2}-2\left(\bar{r}_{\oplus} / R_{\odot}\right) \cos \alpha}},
$$

is used, where $\alpha$ is the angle between $\boldsymbol{R}_{\odot}$ and $\boldsymbol{R}_{\odot}+\overline{\boldsymbol{r}}_{\oplus}$ and $V_{\text {tot }}$ is the total potential at the observed point. Eq. (5) can be expanded in terms of Legendre polynomials as

$$
V_{\mathrm{tot}}=\frac{G M_{\odot}}{R_{\odot}} \sum_{l=0}^{\infty}\left(\frac{\bar{r}_{\oplus}}{R_{\odot}}\right)^{l} P_{l}(\cos \alpha) .
$$

The term $l=0$ is the gravitational potential at the Earth, and the term $l=1$ gives the gravitational field strength at the cen- 
ter of the Earth. Then, the tidal potential is

$$
V_{\text {tide }}(t)=\frac{G M_{\odot}}{R_{\odot}(t)} \sum_{l=2}^{\infty}\left(\frac{\bar{r}_{\oplus}}{R_{\odot}(t)}\right)^{l} P_{l}(\cos \alpha(t)) .
$$

This is eq. (4) in [8]. It should be noted that the time variables on both sides of the equation are the same. In other words, there is no retarded expression $R_{\odot} / c$ in the heliocentric system.

Since the gravimetric observations are made in laboratories on the surface of the Earth, one has to transform the above expression from the heliocentric system to a laboratory system. The laboratory system is chosen in the following way. The positions of the laboratories are labeled by the geographic coordinate system, namely, labeled by their longitudes and latitudes $(\tilde{\theta}, \tilde{\varphi})$, which are fixed (The geographic coordinates are denoted by the letters with over tildes). The position of the Sun is observed optically, assigned with the celestial coordinates, and then mapped to the geographical coordinates $\left(\tilde{\theta}^{\prime}(t), \tilde{\varphi}^{\prime}(t)\right)$, which determine the apparent position of the Sun and depend on time $t$. In terms of geographic coordinates,

$$
\begin{aligned}
V_{\text {tide }}(t)= & \frac{G M_{\odot}}{R_{\odot}(t)} \sum_{l=2}^{\infty}\left(\frac{\bar{r}_{\oplus}}{R_{\odot}(t)}\right)^{l} \frac{4 \pi}{2 l+1} \sum_{m=-l}^{l} Y_{l m}^{*}\left(\tilde{\theta}^{\prime}(t),\right. \\
& \left.\tilde{\varphi}^{\prime}(t)\right) Y_{l m}(\tilde{\theta}, \tilde{\varphi}),
\end{aligned}
$$

where $Y_{l m}^{*}$ is the complex conjugate of $Y_{l m}$. Apparently, the equation seems to have the following explanation: the source at the apparent position at $t$ determines the gravitational potential at the observation point at $t$, or the source at the geometric position at $t_{*}$ determines the potential at the observation point at $t$. But, such an explanation is incorrect because the appearance of the apparent coordinates comes from the coordinate transformation, and the coordinate system with a time-dependent radial coordinate is used. In brief, eq. (8) is just an alternative expression of the standard Newtonian formula. If the substitution of the apparent position of the Sun, $\left(\tilde{\theta}^{\prime}(t), \tilde{\varphi}^{\prime}(t)\right)$, by its geometric position, one will find the discrepancy between the theory and observation by about 500 seconds. On the other hand, if one uses eq. (4) at beginning, then the resulted equation will not be eq. (8) and thus the theory and observation will not be consistent.

Furthermore, consider $S_{2}$ tide (semidiurnal tide produced by the Sun) for simplicity.

$$
\begin{aligned}
\frac{V_{\text {tide }}(t, \tilde{\theta}, \tilde{\varphi})}{g}= & K_{2}\left[P_{2}^{0}\left(\cos \tilde{\theta}^{\prime}\right) P_{2}^{0}(\cos \tilde{\theta})\right. \\
& +\frac{3}{4} \sin \left(2 \tilde{\theta^{\prime}}\right) \sin (2 \tilde{\theta}) \cos \left(\tilde{\varphi}-\tilde{\varphi}^{\prime}(t)\right) \\
& \left.+\frac{3}{8} \sin ^{2} \tilde{\theta}^{\prime} \sin ^{2} \tilde{\theta} \cos \left(2 \tilde{\varphi}-2 \tilde{\varphi}^{\prime}(t)\right)\right]
\end{aligned}
$$

where $g$ is the gravitational acceleration on the surface of the Earth,

$$
K_{2}=\bar{r}_{\oplus} \frac{M_{\odot}}{m_{\oplus}}\left(\frac{\bar{r}_{\oplus}}{R_{\odot}(t)}\right)^{3} .
$$

Note that

$$
\left|\frac{\dot{R}_{\odot}}{R_{\odot}}\right| \ll\left|\dot{\tilde{\theta}^{\prime}}\right| \ll\left|\dot{\dot{\varphi}^{\prime}}\right|=\omega_{\oplus},
$$

where $\omega_{\oplus}$ is the angular velocity of the Earth's spin. Therefore, $R_{\odot}$ and $\tilde{\theta}^{\prime}$ can be treated as constants, and

$$
\tilde{\varphi}^{\prime}(t)=\tilde{\varphi}^{\prime}(0)-\omega_{\oplus} t
$$

Look at the last term of eq. (9),

$$
\frac{3}{8} K_{2} \sin ^{2} \tilde{\theta}^{\prime} \sin ^{2} \tilde{\theta} \cos \left[2 \omega_{\oplus} t+2\left(\tilde{\varphi}-\tilde{\varphi}^{\prime}(0)\right)\right] .
$$

If $\tilde{\varphi}^{\prime}(0)$ is chosen as the prime meridian (i.e. $\tilde{\varphi}^{\prime}(0)=0$ ) and $t$ is reckoned from the instant when the Sun passes the prime meridian, it is the primary astronomical potential (eq. (2) in [3])

$$
\frac{V_{\text {tide }}^{P}(t, \tilde{\theta}, \tilde{\varphi})}{g}=\bar{H} P_{2}^{2}(\cos \tilde{\theta}) \cos (\omega t+2 \tilde{\varphi})
$$

with $\omega=2 \omega_{\oplus}$ and $\bar{H}=\frac{1}{8} K_{2} \sin ^{2} \tilde{\theta}^{\prime}$. The above derivation shows that the phase lags due to the deviation from the spherical, non-rotating, elastic and isotropic (SNREI) model of the Earth is with respect to the standard Newtonian formula. In eqs. (13) or (14), the apparent position of the Sun has been taken into account. It should be remarked that the choice of time-zero and the longitude where the observation station is should be matched together. A mismatch of the time-zero and the longitude will lead to a systematical error. Unfortunately, the problem is not addressed in [1].

In summary, the gravitational field of the Sun is static. There is no propagation problem. The retarded expression comes from the coordinate transformation of the standard Newtonian formula. The conclusion in [1] that gravity propagates with $c$ seems to be drawn based on the misinterpreting of the known formula and the less careful data analysis.

Before the end of the letter, it should be remarked that to check the speed of gravity in GR, the spacetime should be, at least, non-stationary or there exists a non-stationary perturbation in a given stationary spacetime. Moreover, not all non-stationary spacetimes can be used to show gravity propagating at a certain velocity. The most typical example is the Friedmann-Robertson-Walker spacetime. Similarly, in Newtonian theory, only when the source is changing, one may ask whether the gravitational interaction is an instantaneous interaction or propagates at a certain velocity.

This work was supported by the National Natural Science Foundation of China (11275207) and the Oriented Projects of CAS (KJCX2-EW-W01).

1 Tang K Y, Hua C C, Wen W, et al. Observational evidences for the speed of the gravity based on the Earth tide. Chin Sci Bull, 2013, 58: 474-477 
2 Arabelos D. Comparison of Earth-tide parameters over a large latitude difference. Geophys J Int, 2002, 151: 950-956

3 Ray R D, Eanes R J, Lemoine F G. Constraints on energy dissipation in the Earths body tide from satellite tracking and altimetry. Geophys $\mathrm{J}$ Int, 2001, 144: 471-480

4 Sun H P, Xu H Z, Zhou J C, et al. Latest observation results from superconducting gravimeter at station Wuhan and investigation of the ocean tide models (in Chinese). Chin J Geophys (Chin Ver), 2005, 48: 299307
5 Xu J Q, Sun H P, Zhou J C. Experimental detection of the inner core translational triplet. Chin Sci Bull, 2010, 55: 276-283

$6 \mathrm{Xu} \mathrm{J} \mathrm{Q,} \mathrm{Chen} \mathrm{X} \mathrm{D,} \mathrm{Zhou} \mathrm{J} \mathrm{C,} \mathrm{et} \mathrm{al.} \mathrm{Characteristics} \mathrm{of} \mathrm{tidal} \mathrm{grav-}$ ity changes in Lhasa, Tibet, China. Chin Sci Bull, 2012, 57: 25862594

7 Melchior P. The Tides of Planet Earth. New York: Pergamon Press, 1978

8 Agnew D C. Earth tides. In: Herring T, ed. Treatise on Geophysics. Oxford: Elsevier, 2007. 163-195

Open Access This article is distributed under the terms of the Creative Commons Attribution License which permits any use, distribution, and reproduction in any medium, provided the original author(s) and source are credited. 